\title{
Synergy between sulphonamide and trimethoprim in the presence of pus
}

\author{
P. N. EDMUNDS
}

From the Department of Microbiology, Fife Area Laboratory, Scotland, UK

SUMMARY Synergy between sulphadiazine and trimethoprim against Escherichia coli has been demonstrated in culture media not containing lysed horse blood despite the presence of pus or a pus extract which inhibited the action of each drug separately. Synergy may therefore be important where pus is present in vivo.

Darrell et al. (1968) described synergy between sulphafurazole and trimethoprim, using combined sulphonamide (SU) and trimethoprim (TM) or SU and TM discs placed so that their zones of inhibition merged. If synergy was present the combined disc showed a larger zone than the single ones, or distortion of zones cocurred in the now familiar way. Great emphasis was placed from the early days of co-trimoxazole on the clinical importance of synergy of its components, sulphamethoxazole and trimethoprim (Bushby, 1969).

However, there has been a tendency to omit the full three-disc test and even to test only with the combined disc (that is, co-trimoxazole). Doubt was cast by Lewis et al. (1974) and Greenwood and O'Grady (1976) on the importance of synergy both in vitro and in vivo. Emphasis was placed by these authors on the TM rather than the SU component, and this has been interpreted by some as indicating that TM could be used as a single drug in many cases without affecting the clinical result. Great importance has, rightly, been attached to the use of culture medium freed from antagonists by, for example, lysed horse blood (Harper and Cawston, 1945) for carrying out sensitivity testing and especially for demonstration of synergy.

Received for publication 3 August 1977
Little attention has been paid, however, to $\vec{T}$ synergy when pus or other antagonists are present. I present here some instances where synergy has been clearly demonstrated in vitro (at levels of SU and TM attainable in the tissues) in spite of the presence of antagonists in the media.

\section{Methods}

The basic medium described by Macleod (1940) was used, which contains ammonium sulphate, asparagin, glucose, and salts, but with the addition of Difco agar (Cat. No. 0140-01) $1 \cdot 2-1 \cdot 5 \%$. Pus from various lesions, not containing antibiotic, was incorporated in the medium before autoclaving, or an extract was made by diluting the pus 1 in 10 with distilled water, bringing to the boil, centrifuging, and filtering (Whatman grade 113V). The extract was then used instead of distilled water for making up the medium, which was then autoclaved, and plates were poured.

Three strains of Escherichia coli, isolated from infected urine, were selected to give a range of SU sensitivity from highly sensitive to completely resistant by disc test, all being sensitive to TM, and also two strains resistant to TM (Table). SU discs N contained $20 \mu \mathrm{g}$ of sodium sulphadiazine and TM discs $1 \mu \mathrm{g}$ of trimethoprim. Oxoid Diagnostic

Table Strains of Escherichia coli used; sulphonamide and trimethoprim sensitivity and synergy

\begin{tabular}{|c|c|c|c|}
\hline Strain number & Sulphonamide & Trimethoprim & $\begin{array}{l}\text { Synergy demonstrated on } \\
\text { DST lysed blood agar }\end{array}$ \\
\hline $\begin{array}{r}595 \\
23353 \\
23599 \\
16475 \\
15888\end{array}$ & $\begin{array}{l}\text { Highly sensitive } \\
\text { Moderately sensitive } \\
\text { Completely resistant } \\
\text { Highly sensitive } \\
\text { Resistant }\end{array}$ & $\begin{array}{l}\text { Sensitive } \\
\text { Sensitive } \\
\text { Sensitive } \\
\text { Moderately resistant } \\
\text { Resistant }\end{array}$ & $\begin{array}{l}\text { Yes } \\
\text { Yes } \\
\text { No } \\
\text { Yes } \\
\text { No }\end{array}$ \\
\hline
\end{tabular}


Sensitivity Test (DST) agar was used, with the addition of $6 \%$ saponin-lysed horse blood, for sensitivity testing, and the inoculum was an overnight agar culture incubated in air at $37^{\circ} \mathrm{C}$, then diluted in sterile normal saline so as to give semiconfluent growth when flooded on to agar plates. Plates were incubated at $37^{\circ} \mathrm{C}$ in air for $20-44$ hours.

Results were recorded as resistant if colonies were seen next to the disc and sensitive if not. Synergy was shown by a large, swollen zone of inhibition around SU and TM discs placed 6-12 mm apart, much larger than the zones around the discs when placed well apart. Usually no colonies were visible between the discs, but sometimes tiny colonies were seen, though synergy was apparent.

Oxoid DST agar, with lysed horse blood, was tested for para-aminobenzoic acid (PABA) and methionine content by culturing light inocula of a PABA-requiring strain of $E$. coli, AB 3292, and a methionine-requiring strain of $E$. coli, $\mathrm{K} 12$, both diluted as described above. These strains were obtained from Dr. S. G. B. Amyes, now at the Microbiology Department, University of Edinburgh. Luxuriant growth of both strains was obtained. DST agar contains $1 \%$ Proteose peptone and would therefore be expected to include significant amounts of both PABA and methionine. In addition, methionine is present in small amounts in blood.

\section{Results}

A typical example of the picture obtained using a strain highly sensitive to SU is seen in the Figure. The presence of quite large colonies next to the SU and TM discs singly contrasts with their complete absence in the large zone around the two discs placed about $8 \mathrm{~mm}$ apart. The effect of adding pus extract is seen to be particularly dramatic on the SU zone, which was very large on the basic medium, presumably due to its lack of PABA and methionine, as well as thymidine. SU zones were much smaller on the lysed blood agar (Figure). This contains PABA and methionine, as already noted, but not thymidine.

Figure 9-cm agar plate cultures. $A$ and $C$ incubated $20 \mathrm{~h}$, $B 44 h$ at $37^{\circ} \mathrm{C}$ in air.

$B M=$ basic medium; $P E=10 \%$ pus extract. Both

left-hand discs are sulphonamide. In $A$ the zones are so large that they have run together. Both right-hand discs are trimethoprim. $S L B=$ saponin-lysed horse blood Oxoid DST agar.

Note the marked synergy between sulphonamide and trimethoprim in B, which contains pus extract, but resistance to sulphonamide and trimethoprim separately.
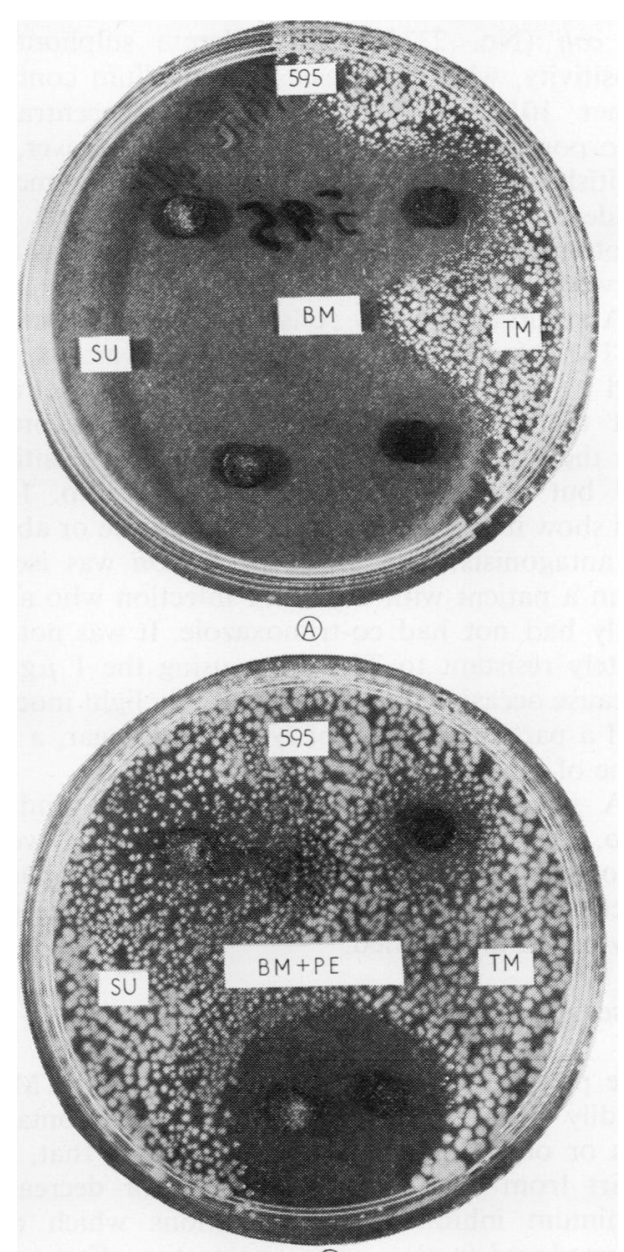

(B)

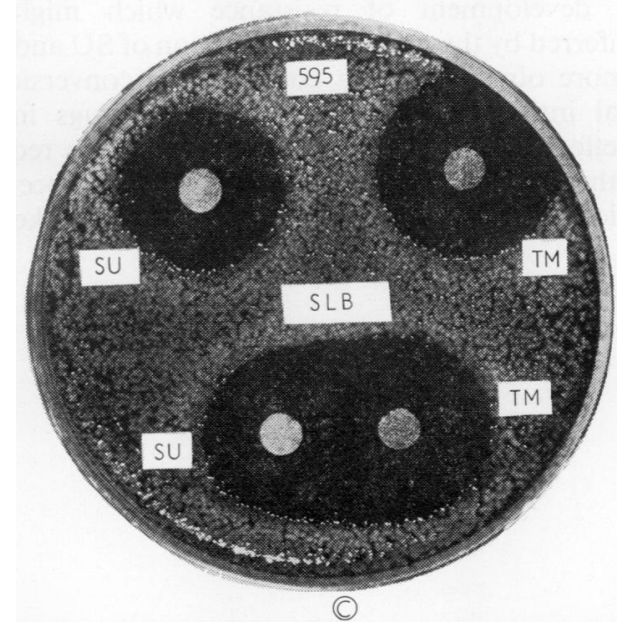


A similar effect was obtained using a strain of E. coli (No. 23353) of moderate sulphonamide sensitivity, when the basic agar medium contained either $10 \%$ or $50 \%$ pus (final concentration), incorporated before autoclaving. However, the whitish opaque appearance of the resulting medium made it impossible to make a sufficiently good photograph for publication due to lack of contrast between colonies and medium.

A strain completely resistant to SU but sensitive to TM (No. 23599) was tested on the various media and invariably failed to show synergy, whether or not there were SU or TM antagonists present. On the other hand, a strain which was sensitive to SU but moderately resistant to TM (No. 16475) did show marked synergy in the presence or absence of antagonists. This strain of $E$. coli was isolated from a patient with a urinary infection who apparently had not had co-trimoxazole. It was not completely resistant to TM when using the $1 \mu \mathrm{g}$ disc, because occasionally, with a specially light inoculum and a particular batch of lysed blood agar, a small zone of inhibition was visible.

A strain completely resistant to SU and TM (No. 15888), isolated from the urine of a woman who had recently had co-trimoxazole without any bacteriological indication, failed to show synergy on any of the media tried.

\section{Discussion}

The presence of synergy between SU and TM was readily demonstrated in culture media containing pus or other antagonists. This suggests that, quite apart from any bactericidal effect or decrease in minimum inhibitory concentrations which might be produced in vivo, or any protective effect against the development of resistance which might be conferred by the use of a combination of SU and TM, a more obvious benefit could be the conversion of total ineffectiveness of the separate drugs in pus to effectiveness when in combination. This requires further research, as a major difficulty has been the collection of enough antibiotic-free pus to make up a reasonable amount of culture medium. The ideal way of preparing and incorporating the pus also remains to be found. Theoretically, one could imitate the effect by adding PABA, methionine, and thymidine, but I suspect that there are other mechanisms of inhibition not yet discovered, and until these have been fully elucidated it will remain a wise precaution to use actual pus to confirm results of obtained by the use of artificial antagonists.

The pus in these experiments was heated either by autoclaving, or boiling and autoclaving. However, $\vec{\omega}$ the likely antagonists PABA, methionine, and thymidine are all heat stable. No doubt it would ? be better to use unheated pus, but I have not yet ${ }_{\vec{\omega}}$ devised a suitable method for incorporating it into is culture medium in a sterile state without the use of $\vec{\sigma}$ heat.

I thank Mr K. Hutchison and Mr M. McDowall for valuable technical assistance, and Dr S. G. B. T Amyes for supplying $\mathrm{PABA}^{-}$and methioninestrains of $E$. coli.

\section{References}

Bushby, S. R. M. (1969). Combined antibacterial action in vitro of trimethoprim and sulphonamides. Postgraduate Medical Journal, 45. Supplement (November), 10-18.

Darrell, J. H., Garrod, L. P., and Waterworth, P. M. (1968). Trimethoprim: laboratory and clinical studies. Journal of Clinical Pathology, 21, 202-209.

Greenwood, D., and O'Grady, F. (1976). Activity and interaction of trimethoprim and sulphamethoxazole against Escherichia coli. Journal of Clinical Pathology, 29, 162-166.

Harper, G. J. and Cawston, W. C. (1945). The in vitro determination of the sulphonamide sensitivity of 8 bacteria.Journal of Pathology and Bacteriology, 57, 59-66.

Lewis, E. L., Anderson, J. D., and Lacey, R. W. (1974). A reappraisal of the antibacterial action of co-trimoxazole in vitro. Journal of Clinical Pathology, 27, 87-91.

Macleod, C. M. (1940). The inhibition of the bacteriostatic action of sulphonamide drugs by substances $\frac{D}{9}$ of animal and bacterial origin. Journal of Experimental Medicine, 72, 217-232. 